\title{
METHODOLOGY OF INTEGRATED MODELING OF HIGH-TEMPERATURE STEEL PROCESSING IN THE ASPECT OF SUPPORTING THE DESIGN OF NEW TECHNOLOGIES
}

\author{
Marcin Hojny, Miroseaw GŁowacki \\ AGH University of Science and Technology, Faculty of Metals Engineering and Industrial Computer Science, \\ Cracow, Poland; e-mail: mhojny@agh.edu.pl
}

\begin{abstract}
The article presents main assumptions of the methodology of integrated modeling of high-temperature steel processing in the aspect of supporting the design of new technologies. The developed solution uses a methodological research capability of modern Gleeble thermomechanical simulators to simulate physical processes, and the benefits of numerical modeling. This allows for restricting the number of expensive experimental tests to the minimum, e.g. by selecting a suitable heating schedule to achieve the desired temperature at the sample section, or getting additional information about the process, eg. estimating the zones with diversified grain growth dynamics, information on local cooling rates at any point within the volume of the sample tested. Mathematical models are original solutions of the developed methodology, such as the thermomechanical model of steel deformation in the semi-solid state, and the multi-scale model of resistance heating coupled with grain growth modelling in the micro scale. The work is supplemented with the main assumptions of the developed mathematical models together with examples of their practical use to support physical simulations.
\end{abstract}

Keywords: finite element method, physical simulation, computer simulation, semi-solid deformation

\section{Introduction}

As the economy keeps growing by implementing innovative technologies, it is necessary to search for new solutions to support decision making processes at the preparation stage and during production. With the present technology development, it is difficult to imagine the operation of a modern industrial plant not benefiting from the application of numerical simulations, either at the engineering or at the production stage. The numerical simulation includes validation and optimisation of products, processes and production tools, practically in every engineering area. On the other hand, more and more common access to modern testing instruments, such as e.g. Gleeble thermomechanical simulators (www.gleeble.com, 2020; Kumar, 2016), computer tomographs (Barciewicz and Ryniewicz, 2018), photogrametric systems (Jiang et al., 2008), thermovision (Rai et al., 2017), combined with commercial computer simulation systems (www.ansys.com, 2019; www.adina.com, 2019), allows us to develop new methodological approaches to designing new and optimising the existing processes. At the same time, original dedicated simulation systems are an alternative to commercial simulation systems (Hojny, 2014). Already at the engineering stage or at the numerical implementation stage, they allow the solution to be optimally adjusted to the specific user requirements (Sommerville, 2011). The physical and numerical modeling of semi-solid steel deformation is an innovative topic regarding very high temperature range deformation processes. Analyzing the available literature, one can find many papers regarding experimental results (Kang and Yoon, 1997; Koç et al., 1996; Kopp et al., 2003) and modelling (Modigell et al., 2004a,b), mainly for non-ferrous metals tests. The first results regarding steel deformation in the semi-solid state were presented in the recent years (Jing et al., 2005; 
Jin Seol et al., 2002; Álvarez Hostos et al., 2018; Shimahara et al., 2006). The situation is caused by a very high level of steel characteristic temperatures (liquidus and solidus) in comparison with non-ferrous metals.

The deformation experimental tests for non-ferrous metals are much easier. The rising abilities of thermomechanical simulators enable steel sample testing and, as a result, both computer simulation and improvement of new rolling technologies, similar to e.g. Iniline Strip Production (Bald et al., 2000; Watari et al., 2004). From technological reasons, a process should be carried out to simplify or eliminate certain operations, thereby reducing energy costs. It is also associated with a favorable impact on the environment, due to reduction of gas emissions. However, in most papers, the necessity of regulated rolling of cast strands is indicated. Therefore, the results of computer simulation or physical simulation of the analyzed process will be useful to control its parameters.

\section{Integrated modeling methodology}

A schematic diagram of the integrated modeling methodology combining the advantages of physical and computer simulation is presented in Fig. 1.

The developed methodology covers two key areas (physical simulation and computer simulation). A Gleeble 3800 thermomechanical simulator performs here a key role (area No. 1), as this is a fundamental tool for simulation of selected manufacturing processes. These simulators are characterized by a resistance sample heating system, and the units for setting deformation are servo-hydraulic devices. It allows high heating rates up to $10000^{\circ} \mathrm{C} / \mathrm{s}$ to be achieved, and a test of sample deformation by compression/tension/torsion to be performed, or selected deformation types to be combined alternately. Figure 2a presents a view of Gleeble 3800 thermomechanical simulator and a view of examples of measurement systems (Fig. 2b) used in the tests. Selection of a suitable simulator measurement system depends on the type of experiment planned and the type of samples applied in the tests. Two types of experiments can be performed with the simulator: i) physical simulation of industrial processes, ii) determination material characteristics.

The idea of physical simulation is to reflect changes in stress states, strains, and temperatures as accurately as possible taking into account non-uniformity of temperature distribution and varying friction conditions, analogous to those occurring in the material processed in industrial conditions.

Gleeble 3800 simulator features a capability of performing an experiment of deformation of a steel sample with a mushy zone occurring in its volume. Integration of the steel strand casting process with subsequent plastic working of steel strands (= Integrated Strip Casting and Rolling) is one of contemporary trends in the steel product manufacturing process development. Solving the most important problems related to the Integrated Strip Casting and Rolling processes will involve the need to determine fundamental high-temperature material characteristics, including curves of high-temperature stress changes versus strain, strain rate and temperature (Hojny, 2018). Rapid state changes, occurring in the steel strand cast and during subsequent mechanical working, require a series of tests of the impact of temperature and strain rate on mechanical properties of the material processed. The capabilities of Gleeble 3800 simulator are now sufficient to carry out a high-temperature experiment, even though it is not amongst the simplest ones. However, difficult interpretation of the findings is one of the fundamental problems. It is not technically possible to carry out experimental tests (e.g. compression or tension) while maintaining a constant temperature and a uniform strain field in the whole area of the sample. Also, it is often difficult to determine the shape of the strain zone itself. For instance, a compressed sample shows strong barrelling of central regions (Fig. 3), while the areas of contact with the tool are virtually not deformed. 


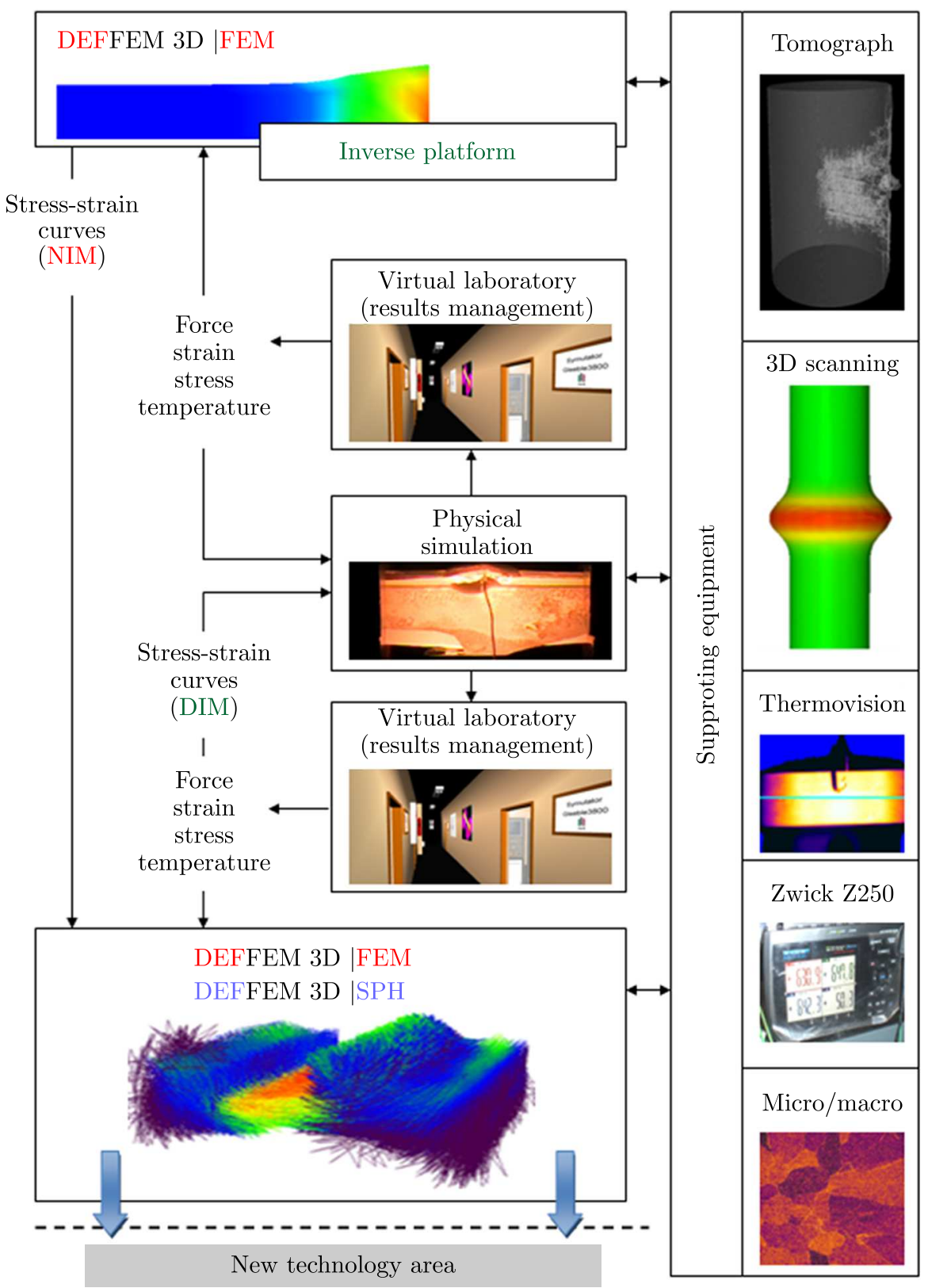

Fig. 1. The scheme of the integrated modeling methodology

(a)

(b)

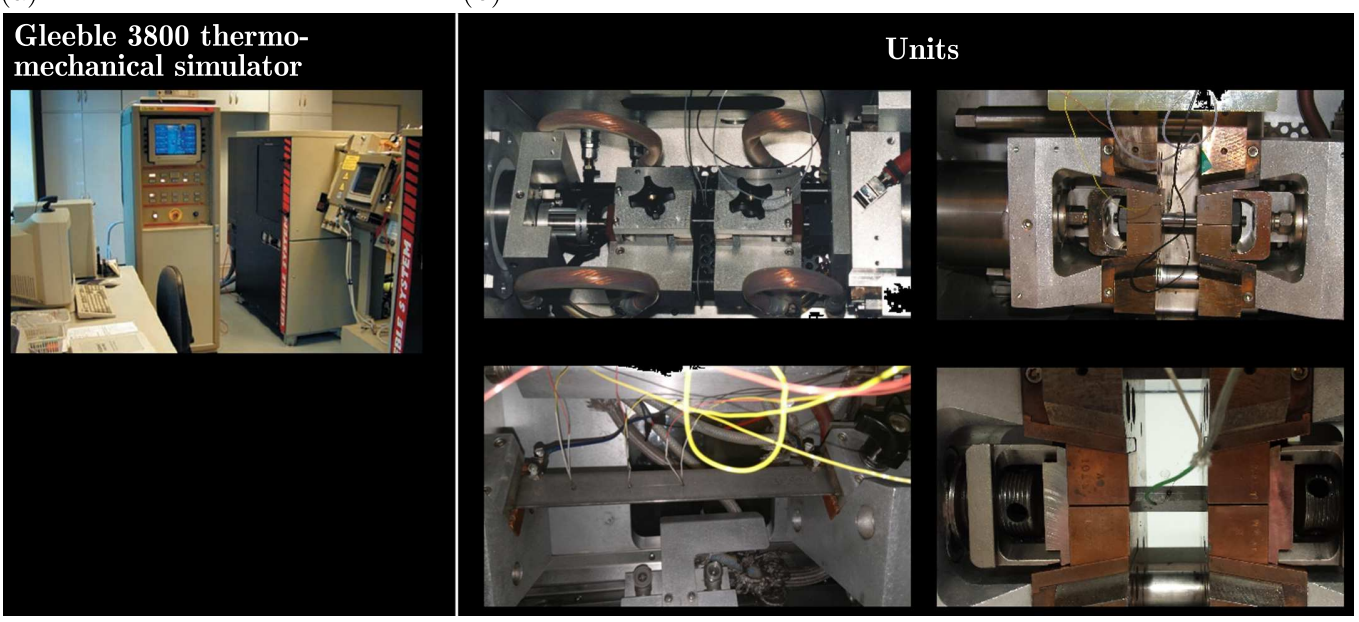

Fig. 2. (a) Gleeble 3800 thermomechanical simulator, (b) view of examples of simulator measurement systems 


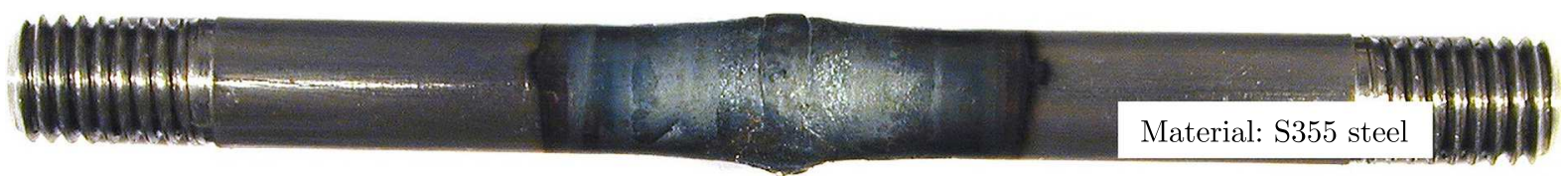

Fig. 3. A sample after completed physical simulation of the "soft-reduction" process

It is caused, inter alia, by a strong dependency of the stress values on temperature, which occurs in temperature ranges near the solidus line of the steel tested. Results of an experiment like this can only be interpreted using computer simulation, e.g. with developed numerical tools implementing unique mathematical models (area No. 2).

\section{Computer aid to high-temperature experiments - original mathematical models and examples of applications}

\subsection{Multiscale model of heating-remelting-cooling}

A standard course of a high-temperature experiment performed with Gleeble 3800 thermomechanical simulator can be divided into three main stages. The first stage is the resistance heating of the sample to the desired nominal temperature in accordance with the assumed heating rates. In the second stage, the sample is held for a certain time at the test nominal temperature in order to stabilize the temperature within the sample volume. At the last stage of the experiment, the sample is deformed (e.g. by compression). The developed numerical model of the resistance heating process is based on the non-stationary solution to the Fourier-Kirchhoff differential equation with an internal voluminal heat source (Hojny, 2018; Hojny et al., 2019)

$$
\nabla^{\mathrm{T}}(\lambda \nabla T)+\left(Q-c_{p} \rho \frac{\partial T}{\partial \tau}\right)=0
$$

where $\tau$ is time, $\rho$ - density, $c_{p}$ - specific heat, $\lambda$ - heat transfer coefficient, $Q$ - rate of heat generation (current flow in the sample).

Equation (3.1) is coupled with the functional model of generating heat resulting from the flow of electric current through the sample (Hojny, 2018; Hojny et al., 2019)

$$
Q=A(\tau)\left[I^{2}(\tau) R(T)\right]
$$

where $I$ is the change of the current intensity versus time $\tau, R$ - change of resistance versus temperature $T, A$ - heating intensity versus time $\tau$.

The boundary conditions were adapted in line with the conditions of Gleeble 3800 simulator module used for the experiments (Fig. 4).

The sample tested made of steel S355 (cubicoidal with length of 124 or $100 \mathrm{~mm}$ and cross-section $10 \times 10$ ) is mounted in two copper grips (Fig. 4). The condition of heat discharge to the environment and to the copper grips is defined as heat fluxes $q_{i}$

$$
q=\alpha_{e}\left(t-t_{i}\right)
$$

where $t_{i}$ is the assumed ambient and tool temperature, $\alpha_{e}$-substitute heat transfer coefficient.

A constant ambient temperature and a tool temperature of $20^{\circ} \mathrm{C}$, and substitute heat transfer coefficients of $120 \mathrm{~W} /\left(\mathrm{m}^{2} \mathrm{~K}\right)$ (exchange of heat with the environment) and $5000 \mathrm{~W} /\left(\mathrm{m}^{2} \mathrm{~K}\right.$ ) (discharge of heat to the tools) were assumed in the calculations. Figure 5 shows an example of the temperature field obtained after heating the sample to a nominal temperature of $1440^{\circ} \mathrm{C}$ (reading of the control thermocouple TC4). As a result of the resistance heating, intensification 


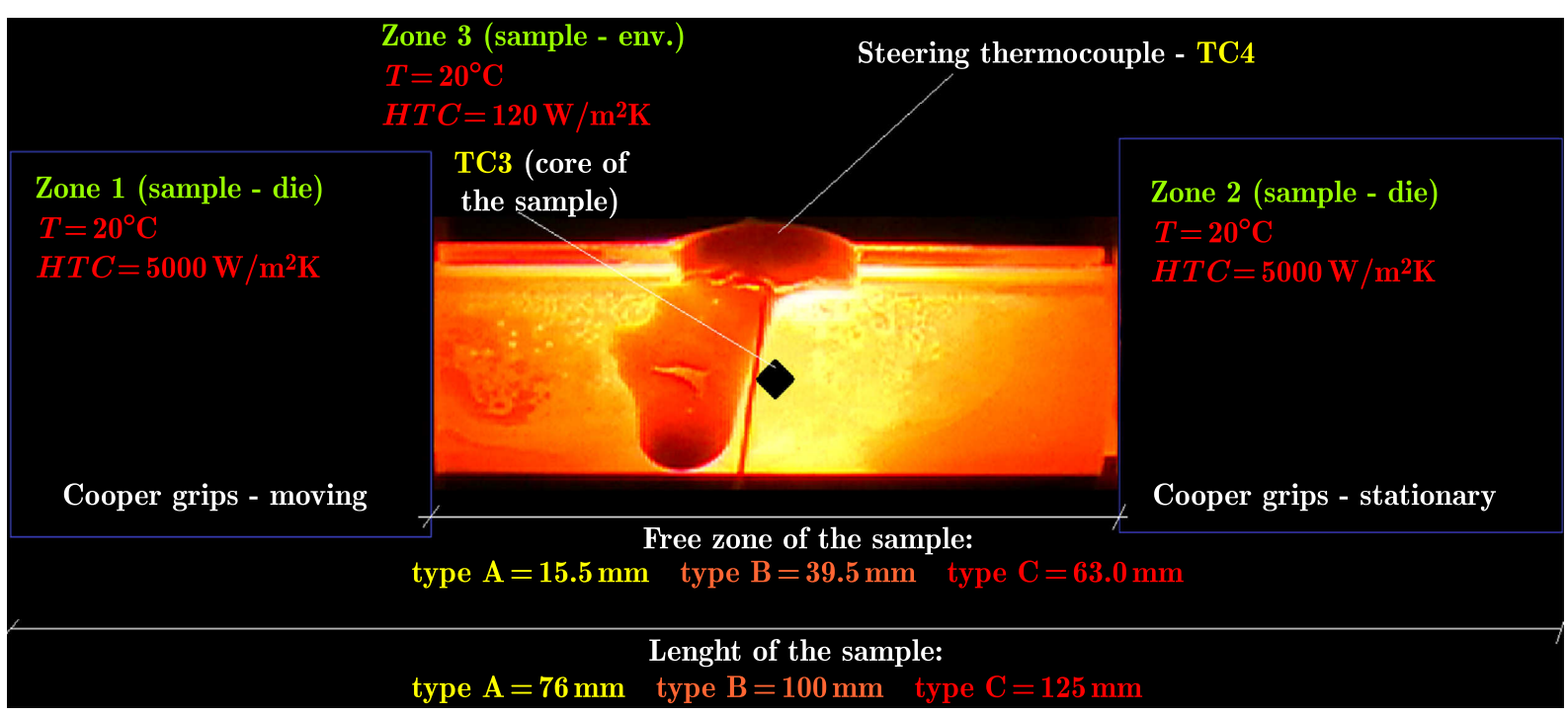

Fig. 4. Diagram of the measurement system with a view of a remelted sample just before the deformation process and with indicated heat transfer zones

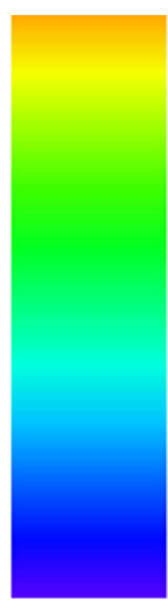

1472.46
1328.53
1184.60
1040.68
896.754
752.828
608.903
464.978
321.052
177.127
33.2012

33.2012

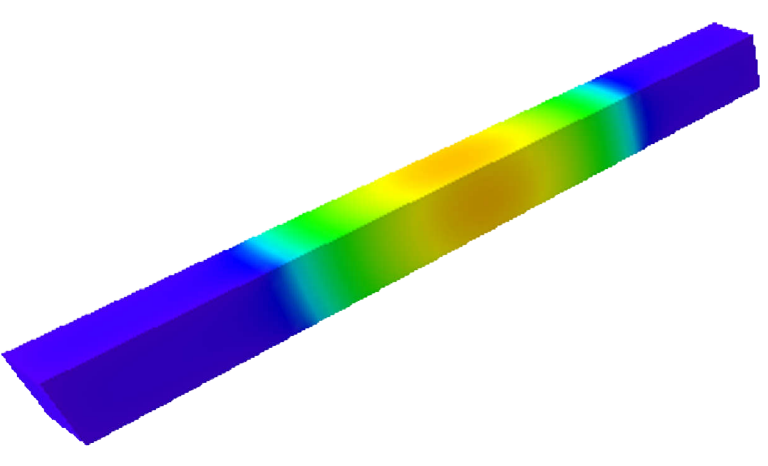

DEFFEM |Post

Fig. 5. The temperature field after heating the sample at a rate of $5^{\circ} \mathrm{C} / \mathrm{s}$ and local sample remelting within the test system of Gleeble 3800 thermomechanical simulator

of the heat emission was at $1 / 2$ of the length of the heated zone of the sample reaching its maximum value of $1472^{\circ} \mathrm{C}$ at the sample core. It makes a difference of $32^{\circ} \mathrm{C}$ between the sample surface (thermocouple TC4) and its core (reading of thermocouple TC3). With reference to the solidus temperature of steel $\mathrm{S} 355$ tested, which is $1465^{\circ} \mathrm{C}$, and the achieved maximum temperature in the sample core of $1472^{\circ} \mathrm{C}$, we can conclude that the zone being a mixture of the solid and liquid phases forms within the sample volume.

During experimental tests (tests repeated three times), the measured temperature of the sample core was $1481.89^{\circ} \mathrm{C}, 1482.45^{\circ} \mathrm{C}$, and $1482.13^{\circ} \mathrm{C}$, respectively. Therefore, the mean difference between the sample surface and its core achieved in the experiment fluctuated within $42^{\circ} \mathrm{C}$. Comparing the results achieved by the experiment and by simulation, one can find that they feature good compatibility (relative error $23 \%$ - cross section temperature gradient). On the basis of the obtained results and the assumptions of the numerical model, we can conclude that the accuracy of the obtained results can be improved by implementing the local boundary condition, which will take into account changes in the substitute heat transfer coefficient as a function of temperature. 
The developed thermal model of the heating/remelting/cooling process may also be coupled with the model of grain micro-growth. The developed solution allows the zone occurrence (within the sample tested volume) to be estimated for diversified dynamics of grain growth. The micromodel is based on the Monte Carlo method, where a local change in energy $E$ is computed in accordance to the equation

$$
E=J \sum_{(i, j)}\left(1-\delta_{i j}\right)
$$

In Eq. (3.4), index $i$ is the number of the subsequent cell, $J$ represents the grain boundary energy, index $j$ is the number of the subsequent neighbour of a specific cell $i$, whereas $\delta$ is the Kronecker delta. It is an original solution of the model in which formation of the mushy zone in the sample core at the stage of local sample remelting is included. If temperature of a specific cell exceeds the critical temperature (most often tantamount to the solidus temperature of the steel tested), melting is simulated by a random assignment of orientation to the sampled cell, different than the states of its neighbours. The classic Monte Carlo algorithm modified this way, comprehensively includes simulation of the heating process combined with local remelting followed by free cooling within Gleeble simulator tool system. It allows three mechanisms of formation and creation of new grains to be included in the model: free grain growth in the not remelted zone, epitaxial grain growth at the grain boundary of the remelted zone, formation of new grains in the remelted zone and their free growth. Figure 6 presents examples of the macro-structure at the longitudinal section of the sample for selected stages of comprehensive simulation of the heating combined with remelting followed by free cooling within the simulator tool system. The calculations were performed for $1 / 2$ of the sample length. Analysing the obtained results, one may observe the occurrence of zones with diversified grain growth at the longitudinal section (the final stage of heating). The diversified dynamics of grain growth in individual areas of the sample results from the obtained large temperature gradients at the longitudinal and transverse sections of the sample tested. To include local temperature changes, a change in the cell state is accepted with the probability of $P$

$$
P=\left\{\begin{array}{lll}
B_{m}(T) \exp \left(-\frac{\Delta E}{k T}\right) & \text { for } & \Delta E>0 \\
B_{m}(T) & \text { for } & \Delta E \leqslant 0
\end{array}\right.
$$

where $k T$ is a parameter equal to $0.4, \Delta E$ - energy of grain orientation change, $B_{m}(T)$ - scaling function taking into account the impact of local temperature value on the value of probability.

At the initial stage of the remelting process, one may observe formation of the mushy zone in which the volume grows as the nominal heating temperature increases. During free cooling within the simulator tool system, new grains form within the obtained mushy zone, there is also an epitaxial growth at the boundary of the remelted grains. As a result of the obtained high cooling rates within the simulator tool system, the final macrostructure is finer than the one obtained at the end of the heating process. More details and verification test results for the developed multi-scale model are presented in paper (Hojny et al., 2019).

\subsection{Thermo-mechanical model of deformation in the semi-solid state}

The process of sample deformation, e.g. by compression, is the last stage of a high-temperature experiment. In order to include in the numerical model the impact of the mushy zone forming within the sample volume on the shape of the deformation zone obtained, a 


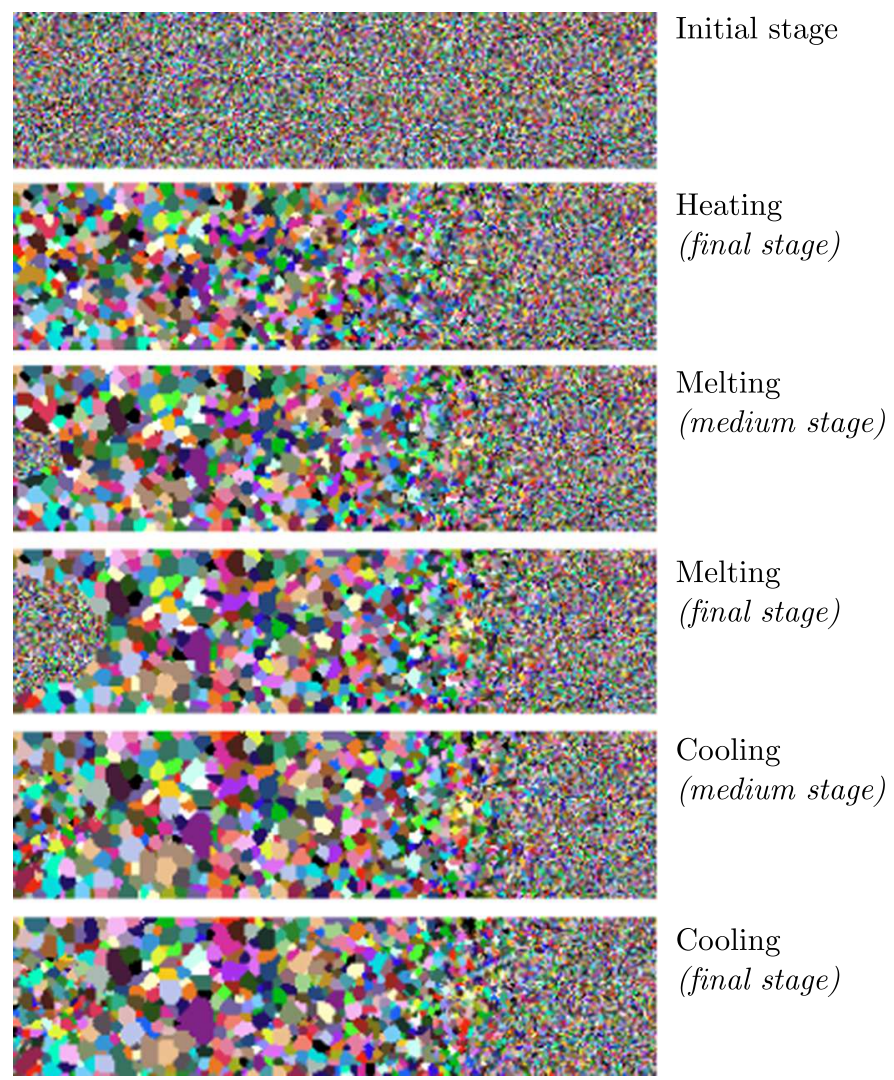

Fig. 6. Macrostructure at the longitudinal section of the sample for selected stages of the heating process combined with remelting and free cooling within Gleeble 3800 simulator tool system

modified rigid-plastic solution was proposed (Hojny, 2018). The classic solution is based on the optimisation of the power functional $F$

$$
F=\dot{W}_{\sigma}+\dot{W}_{\lambda}+\dot{W}_{t}
$$

The right hand side of functional (3.6) consists of three factors. The first one is $\dot{W}_{\sigma}$, or the plastic deformation power, the second one $\dot{W}_{\lambda}$ is the power to change the volume, the last factor is $\dot{W}_{t}$, or the friction force power. In the proposed solution, the factor being the friction force power was omitted in the functional $F$. This assumption arises from the fact that during the high-temperature experiments, the samples were permanently fixed in the tools of the Gleeble simulator system. In addition, whereas the mushy zone forming in the sample volume featured a variable density caused by an on-going change in the state of aggregation, the classic constant volume condition was replaced by the condition of variable density, in accordance with the equation

$$
\dot{W}_{\lambda}=\int_{V} \lambda\left(\frac{\partial v_{x}}{\partial x}+\frac{\partial v_{y}}{\partial y}+\frac{\partial v_{z}}{\partial z}-\frac{1}{\rho} \frac{\partial \rho}{\partial T}\right) d V
$$

The basis for the optimizing solution of functional (3.6), taking into account the condition of variable density, is the velocity field $\left(v_{x}, v_{y}, v_{z}\right)$ determined by an appropriate system of velocity functions in the sample tested volume concerned.

High-temperature curves including changes of stress versus strain, temperature and strain rate (optionally, liquid phase fraction) are inseparable components of the proposed solution. They have a substantial impact on the accuracy of prediction of the shape and size of the deformation zone. The monograph (Hojny, 2018) presents two methodological solutions (NIM - 
Numerical Identification Methodology and DIM - Direct Identification Methodology) utilizing the capabilities of Gleeble simulators and the designed numerical tools for developing a rheological model of the steel tested. Figure 7 presents an example of a high-temperature stress-strain curve for a temperature of $1450^{\circ} \mathrm{C}$ and a stroke rate of $0.05 \mathrm{~s}^{-1}$.

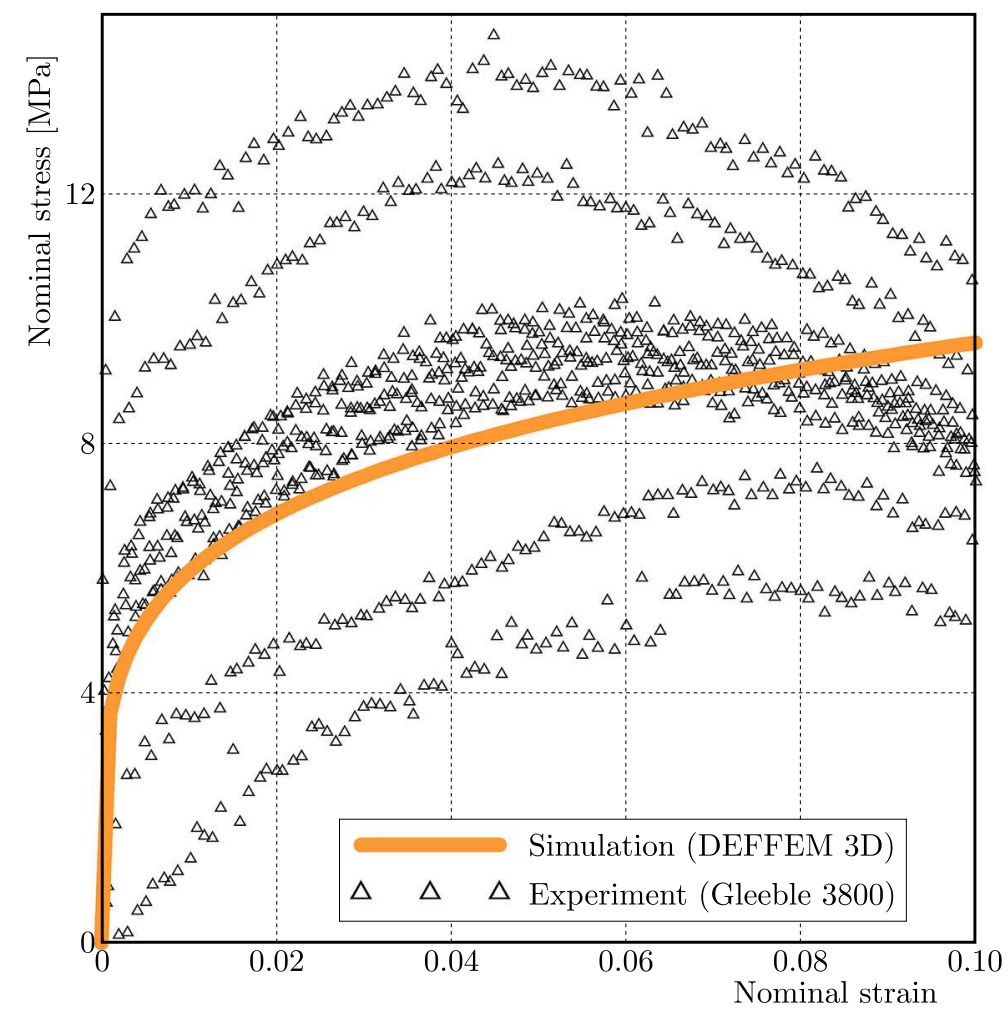

Fig. 7. An example of a high-temperature stress-strain curve (steel grade S355, Direct Identification Methodology)

Figure 8 presents the strain intensity field in the sample deformed at a temperature of $1435^{\circ} \mathrm{C}$ at a stroke rate equal to $1 \mathrm{~mm} / \mathrm{s}$ and the stroke $5 \mathrm{~mm}$. The strain accumulation and the zone subject to deformation occur at $1 / 2$ of the length of the sample zone deformed, reaching their maximum value at the core sample equal to 0.72 .
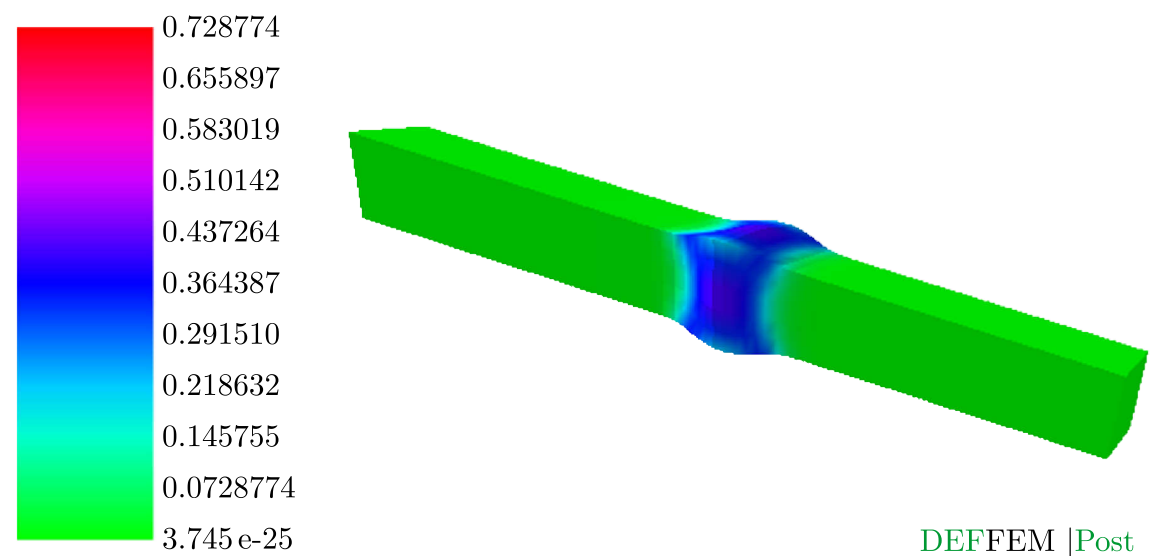

DEFFEM |Post

Fig. 8. The strain intensity field after deforming the sample within Gleeble 3800 thermomechanical simulator system

Atos Triple Scan 3D scanning system from GOM was used to verify correctness of the prediction of the shape and size of the deformation zone. The obtained digital sample shape was 
then compared with the sample geometry obtained by simulation. Two criteria were assumed for comparison, i.e. measurement of cross-section of the deformation zone and length of the deformed zone. In the presented case, the obtained values of the relative error for both criteria were $16 \%$ and $7 \%$, respectively.

\section{Examples of applications of the integrated modeling methodology to aid engineering new processes}

The developed integrated modeling methodology was utilized in practice during engineering work to develop and implement the process of hot forming of a reinforcing component of an intermediary casing, directing the air flow in a jet engine (Pieja et al., 2017). During the research and development work, a 3D model of resistance heating was adapted to industrial conditions. The objective of numerical simulations was to estimate and select characteristics of current intensity changes versus time to achieve the assumed temperature of the plate heated (material: Inconel 719) in compliance with the process engineering assumptions. Figure 9 presents an example of the temperature field for heating a metal plate in Gleeble 3800 thermomechanical simulator system. In the simulation, the plate was resistance heated to a nominal temperature of $1000^{\circ} \mathrm{C}$. As a result of the numerical simulation, the maximum temperature of $987^{\circ} \mathrm{C}$ was achieved. On the other hand, the maximum temperature recorded during physical simulation was $999^{\circ} \mathrm{C}$, which yielded a relative error between the both values of $1.2 \%$.
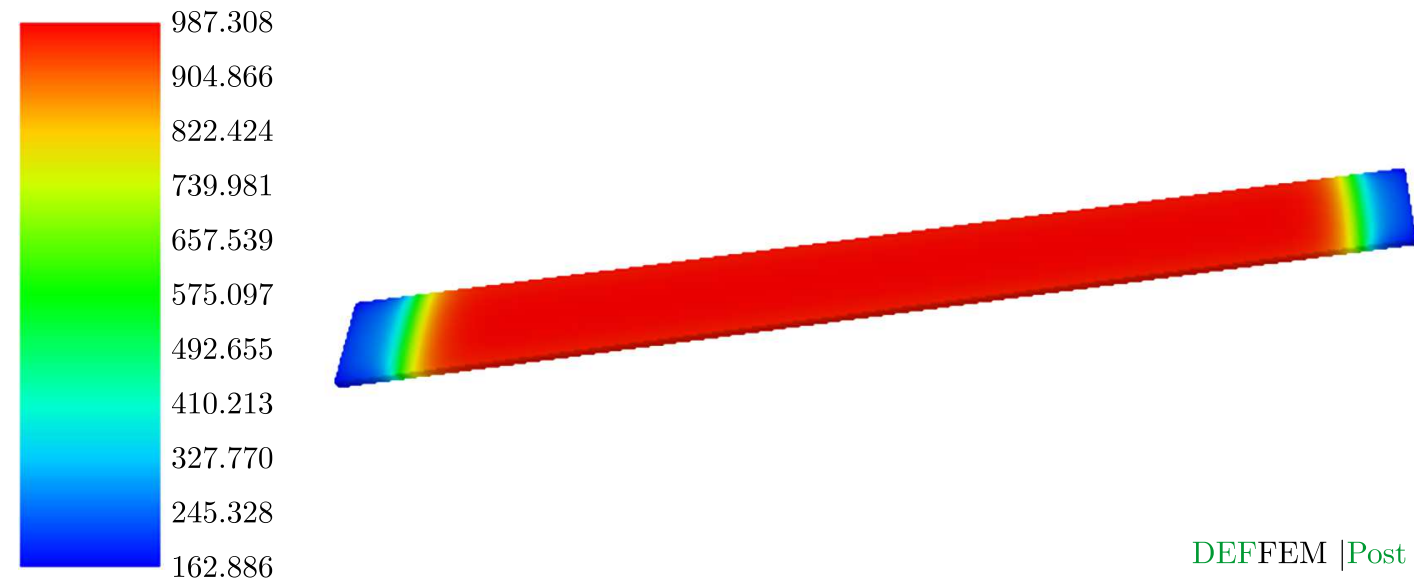

Fig. 9. Temperature field after plate resistance heating in Gleeble 3800 simulator system

Based on the data from physical simulations, simulations of the resistance heating of a metal plate were performed in accordance with the industrial conditions. Figure 10 presents an example of a temperature field within a metal plate heated by resistance. The maximum computed temperature value was $173^{\circ} \mathrm{C}$. During the completed industrial tests, the maximum temperature value achieved was $181^{\circ} \mathrm{C}$, which yielded a relative error between the both values of $4.4 \%$.

\section{Summary}

The article presents the main assumptions of the developed methodology of integrated modeling combining the advantages of physical and computer simulation in the aspect of supporting high-temperature experiments. The developed mathematical models are unique achievements of the proposed solution. The first model is a multi-scale model of heating/remelting steel samples, 

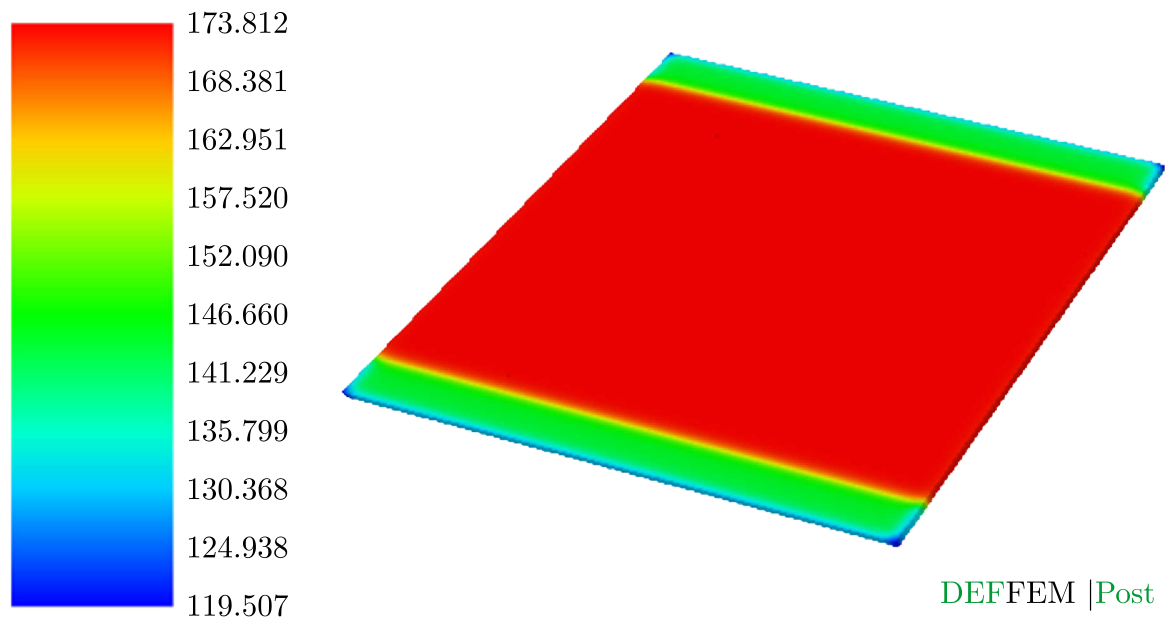

DEFFEM |Post

Fig. 10. Temperature field after resistance heating of a metal plate in industrial conditions (material: Inconel 718 , test temperature $181^{\circ} \mathrm{C}$ )

which combines with the model of grain micro-growth, enables zones with diversified dynamics of the grain growth within the sample volume to be estimated. The possibility of comprehensive simulation of the heating-remelting-cooling process taking into account formation of a mushy zone at the micro-model level (remelting stage) is a specific feature of the solution. The model of deformation of steel samples with a mixture of liquid and solid phase occurring in their volume is another original solution. In order to include the mushy zone at the mechanical model level, the classic rigid-plastic solution was changed by modifying the power functional replacing the condition of constant volume by the condition of variable density. The model assumptions and the correctness of numerical implementation was verified in practice during a cycle of hightemperature experiments with the simulator. The obtained simulation results featured a good compatibility with the results obtained experimentally. Taking into account the utilitarian potential of the proposed solutions, further research and development work was focused on the development of models based on meshless methods. It will allow us to include local flows of solidifying steel during the deformation process, or identify discontinuity areas caused by shrinkage of the solidifying metal. Finally, it will result in a reduction of costly experimental tests to the minimum, and in additional information on the process simulated both physically and numerically.

\section{Acknowledgment}

The research work has been supported by the Polish National Science Centre, decision number: DEC-2011/03/D/ST8/04041.

\section{References}

1. Álvarez Hostos J.C., Bencomo A.D., Puchi Cabrera E.S., Guérin J.-D., Dubar L., 2018, Modeling the viscoplastic flow behavior of a $20 \mathrm{MnCr} 5$ steel grade deformed under hot-working conditions, employing a meshless technique, International Journal of Plasticity, 103, 119-142

2. BALd W., 2000, Innovative technologies for strip production, Steel Times International, 24, 16-19

3. BARCiewicz M., Ryniewicz A., 2018, The application of computed tomography in the automotive world - how industrial CT works, Technical Transactions, 9, 181-188

4. Hojny M., 2014, Designing Dedicated Simulation Systems of Steel Deformation in the Semi-Solid State (in Polish), Wzorek, Kraków 
5. Hojny M., 2018, Modeling of Steel Deformation in the Semi-Solid State, Springer, Switzerland

6. Hojny M., GŁowacki M., BaŁa P., Bednarczyk W., Zalecki W., 2019, A multiscale model of heating- remelting-cooling in the Gleeble 3800 thermomechanical simulator system, Archives of Metallurgy and Materials, 64, 1, 401-412

7. http://www.gleeble.com (access 10.01.2020)

8. http://www.ansys.com (access 30.10.2019)

9. http://www.adina.com (access 30.10.2019)

10. Jiang R., JÁuregui D.V., White K.R., 2008, Close-range photogrammetry applications in bridge measurement: Literature review, Measurement, 41, 823-834

11. Jin Seol D., Он K.H., Cho J.W., Lee J.-E., Yoon U.-S., 2002, Phase-field modelling of the thermomechanical properties of carbon steels, Acta Materialia, 50, 2259-2268

12. Jing Y.L., Sumio S., Jun Y., 2005, Microstructural evolution and flow stress of semi-solid type 304 stainless steel, Journal of Materials Processing Technology, 161, 396-406

13. Kang C.G., Yoon J.H., 1997, A finite-element analysis on the upsetting process of semi-solid aluminum material, Journal of Materials Processing Technology, 66, 76-84

14. Koç M., Vazques V., Witulski T., Altan T., 1996, Application of the finite element method to predict material flow and defects in the semi-solid forging of A356 aluminum alloys, Journal of Materials Processing Technology, 59, 106-112

15. Kopp R., Choi J., Neudenberger D., 2003, Simple compression test and simulation of an $\mathrm{Sn}-15 \% \mathrm{~Pb}$ alloy in the semi-solid state, Journal of Materials Processing Technology, 135, 317-323

16. Kumar V., 2016, Thermo-mechanical simulation using Gleeble system - advantages and limitations, Journal of Metallurgy and Materials Science, 58, 1, 81-88

17. Modigell M., Hufschmidt M., Petera J., 2004a, Two-phase simulations as a development tool for thixoforming processes, Steel Research International, 75, 513-518

18. Modigell M., Pape L., Hufschmidt M., 2004b, The rheological behaviour of metallic suspensions, Steel Research International, 75, 506-512

19. Pieja T., Malinowski T., Hojny M., Trzepieciński T., Nowotyńska I., 2017, Numerical analysis of cooling system in warm metal forming process, Proceedings of 26th International Conference on Metallurgy and Materials, 261-266

20. Rai M., Maity T., Yadav R.K., 2017, Thermal imaging system and its real time applications: a survey, Journal of Engineering Technology, 6, 2, 290-303

21. Shimahara H., BaAdjou R., Kopp R., Hirt G., 2006, Investigation of flow behaviour and microstructure on X210CrW12 steel in semi-solid state, Splid State Phenomena, 116, 189-192

22. Sommerville I., 2011, Software Engineering, Addison-Wesley, Boston

23. Watari H., Davey K., Rasgado M.T., Haga T., Izawa S., 2004, Semi-solid manufacturing process of magnesium alloys by twin-roll casting, Journal of Materials Processing Technology, 156, 1662-1667 